\title{
Complex Strain Paths in Polycrystalline Copper:
}

\section{Microstructural Aspects}

\author{
M.F. Vieira ${ }^{\mathrm{a}}$, J.V. Fernandes ${ }^{\mathrm{b}}$ \\ a Instituto de Materiais / Departamento de Engenharia Metalúrgica e Materiais, FEUP \\ Rua dos Bragas, 4099 Porto Codex, Portugal \\ ${ }^{\mathrm{b}}$ Departamento de Engenharia Mecânica, FCTUC, Universidade de Coimbra \\ Pólo 2 da Univ. Coimbra, Pinhal de Marrocos, 3000 Coimbra, Portugal
}

Received: August 15, 1998; Revised: March 30, 1999

\begin{abstract}
Microstructural aspects of polycrystalline copper sheets subjected to complex strain paths were analysed in this work. Dislocation structures developed during the strain paths (rolling and tension) and the evolution of this microstructure during reloading have been studied. The active slip systems developed in each strain path were used to explain the microstructural evolution. The heterogeneous surface deformation observed on polished tensile specimens prestrained in rolling was also analysed. The structural aspects are related with the mechanical behaviour of the material, namely with the increase in yield stress in reloading, the work hardening evolution and the premature occurrence of plastic instability for some prestrain values.
\end{abstract}

Keywords: complex strain paths, microstructural aspects, plastic instability

\section{Introduction}

In complex strain paths, the mechanical behaviour observed in reloading diverges from that typical of a monotonic deformation. Most cases concern the behaviour where a relatively high initial flow stress (compared with the stress at the same equivalent strain in the monotonic path) is followed by a low work-hardening rate and by a reduction in the total plastic homogeneous deformation ${ }^{1-3}$.

Several authors have presented details of microstructural features during various complex strain paths of ductile materials ${ }^{4-9}$, for example . After a change of strain path, two main cases of microstructural modifications have been observed, depending on the material, grain size, and type of path change. In some cases, microbands appear inside the grains at the first stages of deformation during reloading $^{4-6}$; in other cases, dislocation arrangements evolve gradually during the second path, and at large strains the dislocation structures tend to be similar to the substructure typical of the new deformation mode ${ }^{7-9}$.

The aims of the present research were, first, to examine the mechanical behaviour and the microstructural evolution of copper sheets during sequences of orthogonal rolling-tension tests and secondly, to discuss connections between slip behaviour inside the grains and the surface strain distribution at the beginning of reloading.

\section{Experimental}

Oxygen-free high purity copper $(99.95 \% \mathrm{Cu})$ sheet, 1 $\mathrm{mm}$ thick, previously cold-rolled and annealed, was used in this research. The grains were equiaxed with a $7 \mu \mathrm{m}$ size.

Sequences of two strain paths were performed at room temperature. The prestrain path was a unidirectional rolling. Square samples $\left(10^{4} \mathrm{~mm}^{2}\right)$ were deformed up to von Mises equivalent strains between 0 and 0.26 . The tensile test specimens had a parallel length of $60 \mathrm{~mm}$ and a width of $10 \mathrm{~mm}$. The tensile axis was normal to the rolling direction. An extensometer with a gauge length of $50 \mathrm{~mm}$ was used to measure displacement and a computer to give true stress-true strain curves processed both load and displacement.

To characterise strain distribution during the second path in tension, some of the tensile specimens were carefully polished after prestraining. The specimens were electropolished by immersion in a solution containing $250 \mathrm{~mL}$ orthophosphoric acid, $250 \mathrm{~mL}$ ethanol, $500 \mathrm{~mL}$ distilled water and $3 \mathrm{~g}$ urea and using a current density of 8 $\mathrm{mA} / \mathrm{mm}^{2}$. The microstructure of the deformed sample was observed through light and transmission electron microscopy $(100 \mathrm{kV})$. The observation plane was the sheet plane. Sample preparation has been described elsewhere?.

\footnotetext{
a e-mail: mvieira@fe.up.pt
} 


\section{Results}

\subsection{Mechanical behaviour}

Examples of true stress-true strain curves are shown in Fig. 1. These curves present a macroscopic reloading yield stress greater than the stress reached at the same strain along the reference curve (without prestrain) and the slope of the curves of prestrain samples is lower than the slope of the monotonic curve, at the same equivalent strain. From the analysis of Fig. 1, it can be also concluded that flow localisation appears immediately after the yield stress for prestrain value higher than $\varepsilon_{\mathrm{p}}>0.10$.

Similar behaviour has been already reported for copper $^{10}$. However, the nominal stress $(\Sigma)$ - nominal strain (e) curves (Fig. 2) presents a behaviour not commonly observed in tension: samples with prestrain values of 0.08 and 0.10 exhibited two load peaks; a sharp peak near yielding and a broad peak at higher strain. After the sharp peak, the slope of the corresponding true stress - true strain curves are greater than zero which means that the deformation is not of the Luders band type.

\subsection{Microstructural observations}

\section{a) Dislocation structure}

TEM observations, after deformation in rolling, have shown that the dislocation microstructure in the sheet plane consists of a loose cell structure (Fig. 3) up to strain values of 0.26 , independent of crystallographic orientation. In tension, the dislocation cell structure is better defined than in rolling: the dislocation walls thickness and the dislocation density in the cell interior are smaller, for the same strain value. Besides, two intersecting families of parallel dislocation walls are visible in some grains with particular orientation (Fig. 4).

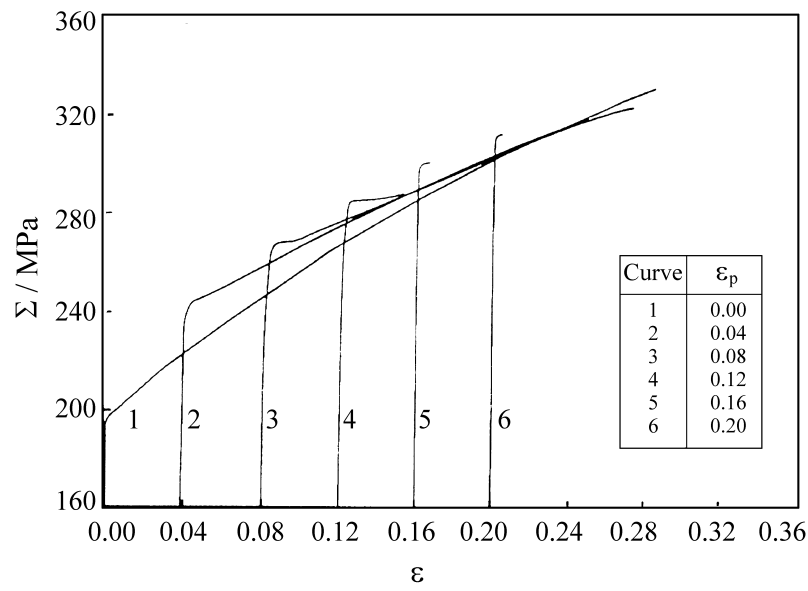

Figure 1. Examples of true stress $(\sigma)$ - true strain $(\varepsilon)$ curves in tension of samples prestrained in rolling. The curve without prestrain $\left(\varepsilon_{p}=0\right)$ is also shown.

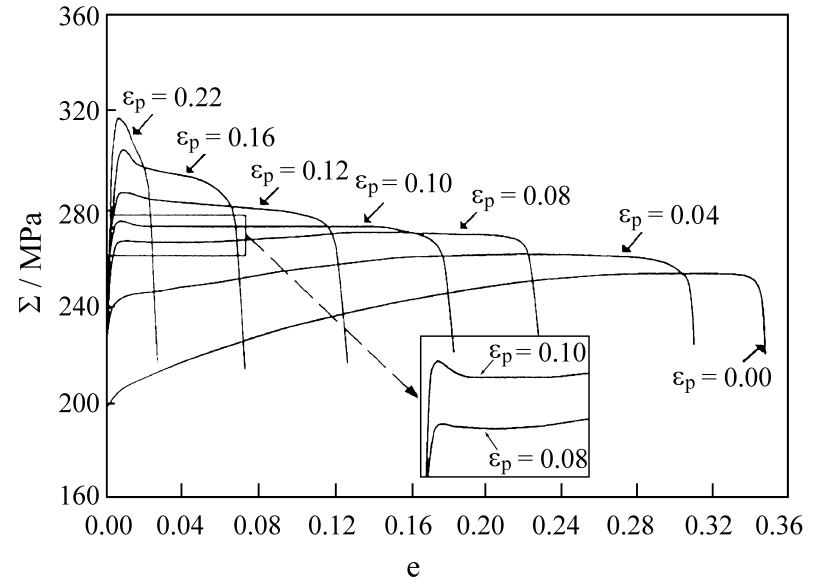

Figure 2. Examples of nominal stress $(\Sigma)$ - nominal strain (e) curves in tension of samples prestrained in rolling. The curve without prestrain $\left(\varepsilon_{p}=0\right)$ is also shown.

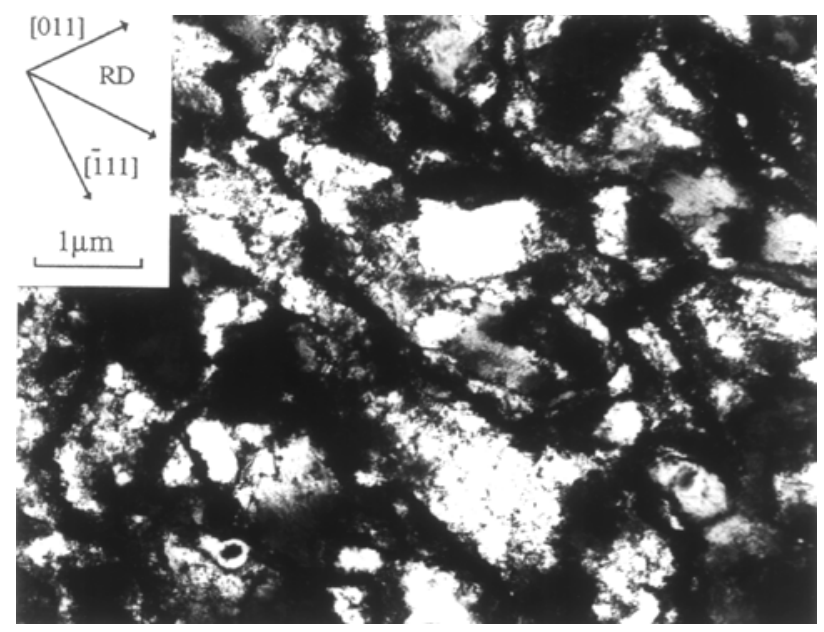

Figure 3. TEM observation of closed cell structure after a strain amount in rolling equal to 0.26 in a grain with $<112>$ axis normal to the sheet plane ( $\mathrm{RD}$, rolling direction).

At the beginning of reloading, the dislocation microstructure was still more disorganised than in rolling (Fig. $5)$. When it was possible to attain large deformation in the subsequent tension, two intersecting families of parallel straight dislocation walls were observed a few times in the macroscopic strain localisation region (Fig. 6), as in tension without prestrain.

\section{b) Surface structure}

During the reloading in tension, the slip lines were observed through an optical microscope by differential interference contrast. In order to follow the evolution of slip line pattern during deformation, observations were performed after several strain values; also several prestrain values (including zero) were used. One slip line system (set of parallel slip lines in a grain) was observed in most of the grains (Fig. 7); in some grains other families of slip lines are noted, but frequently in restricted areas. These obser- 
vations are independent on the prestrain value, the strain amount in tension and on the observed region.

Regions of localised deformation at the grain level were not observed. The slip lines do not cross the grain boundaries; these did not show offsets or steps, even in the necking region of the highest prestrained samples.

\subsection{Macroscopic strain distribution}

Photographs were taken during the tensile tests of samples specially prepared by polishing to permit the evolution of the deformed region to be examined. This evolution depends mainly on the prestrain value. For low prestrain values (0.04 and below, including the samples without prestrain), the deformed (bright) region covers the entire specimen and can be observed after a deformation of about 0.03 . When the prestrain value is in the range 0.06 to 0.10 ,

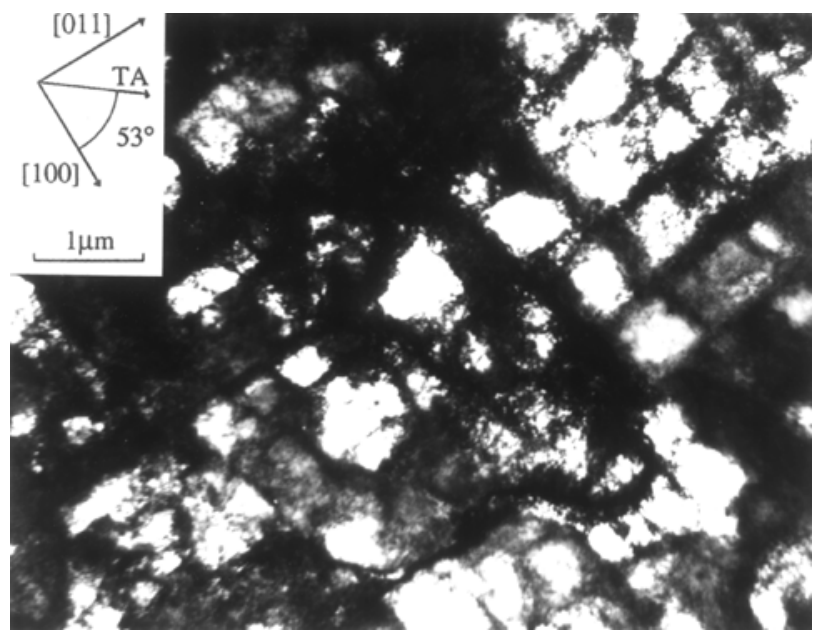

Figure 4. TEM observation of closed parallelogram cells developed in a grain with $<1 \quad 10>$ axis normal to the sheet plane after a strain amount equal to 0.25 (TA, tensile axis).

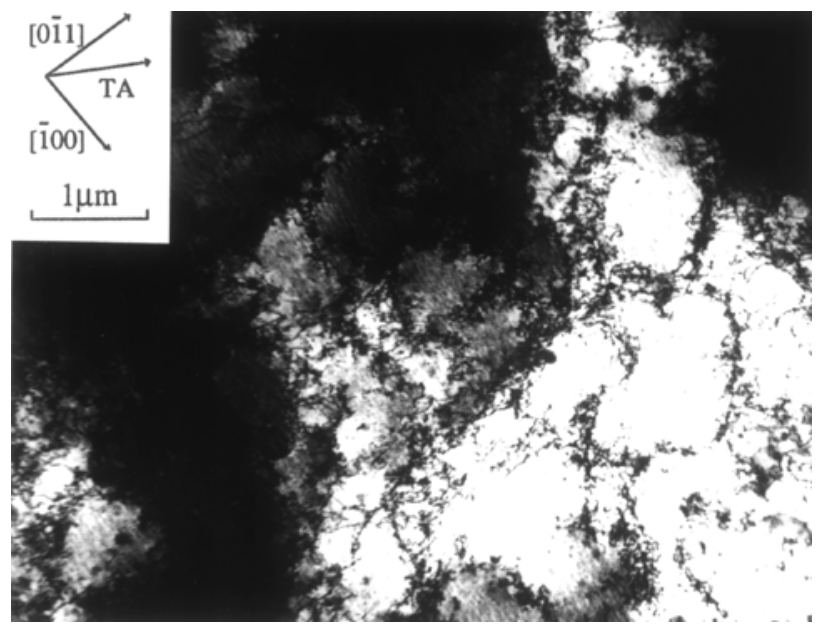

Figure 5. TEM observation of unorganized dislocation structure, developed during the second path in tension (strain approximately equal to 0.02 ) in a grain with $<110>$ axis normal to the sheet plane. The prestrain value in rolling is equal to 0.16 (TA, tensile axis). one region where deformation begins is clearly observed; this region spreads out with deformation, but sometimes rupture arises before the whole sample has been seen to deform (Fig. 8(a)). For prestrain values where only one peak load appears immediately after yielding (highest prestrain values), the observed localised region has a fairly defined profile from the start of deformation and spreads out just a little before rupture (Fig. 8(b)).

\section{Discussion}

The observed increase in yield stress after path change can be related to the physical mechanisms that occur during subsequent deformation. The latent hardening effect plays an important role in this behaviour, which can be analysed by investigating the nature of the active slip systems during the two successive strain path ${ }^{6}$. Assuming that the intra-

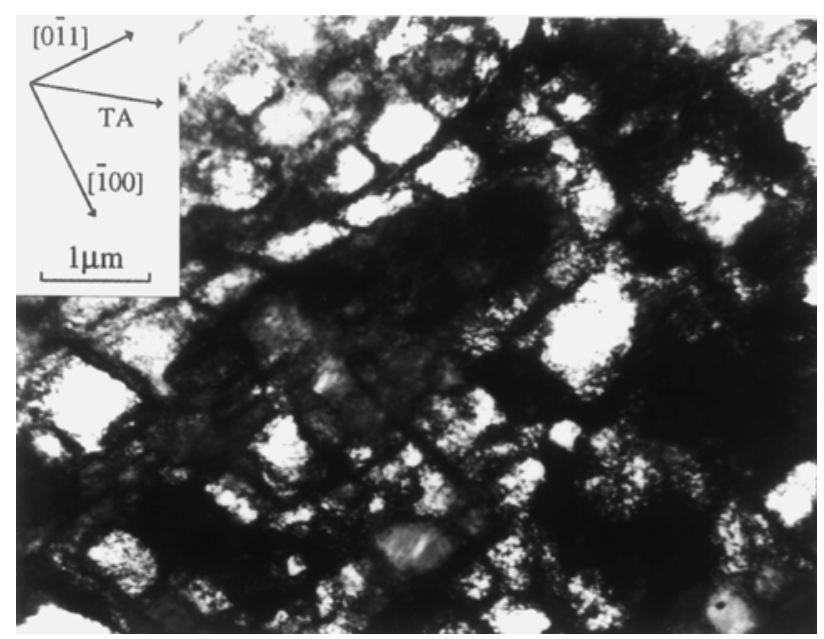

Figure 6. TEM observation of closed parallelogram cells developed near the rupture line during the second deformation in a grain with $\langle 110>$ axis normal to the sheet plane. The prestrain value in rolling is equal to 0.16 (TA, tensile axis).

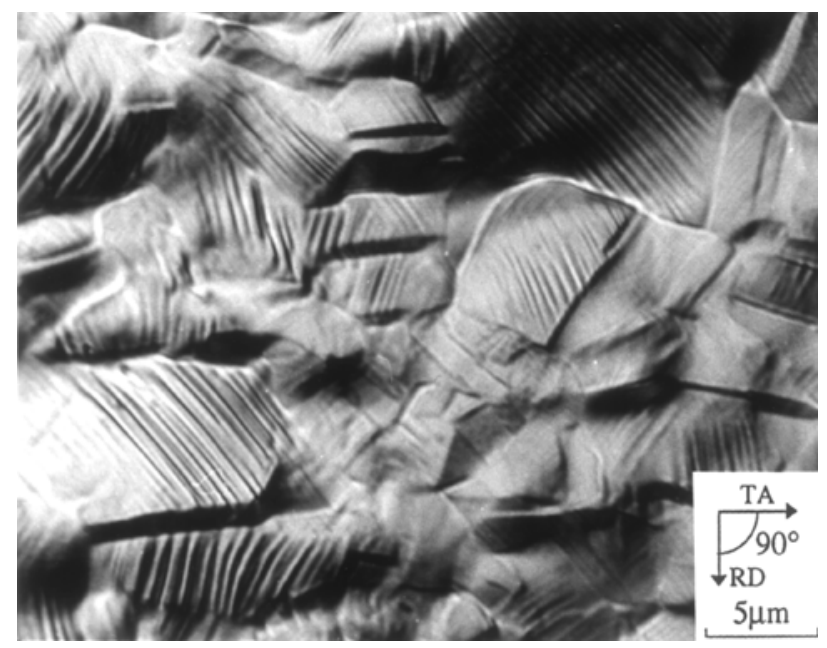

Figure 7. Optical observation of slip lines, developed during the second deformation in the macroscopic localized region (after rupture). The prestrain value is equal to 0.22 . 
granular behaviour follows the Taylor model ${ }^{11}$, the sets of possible active slip systems during the two successive paths have been estimated for the three main components of the texture in the sheets. The possible active slip systems, presented in Table 1, are listed when they account for more
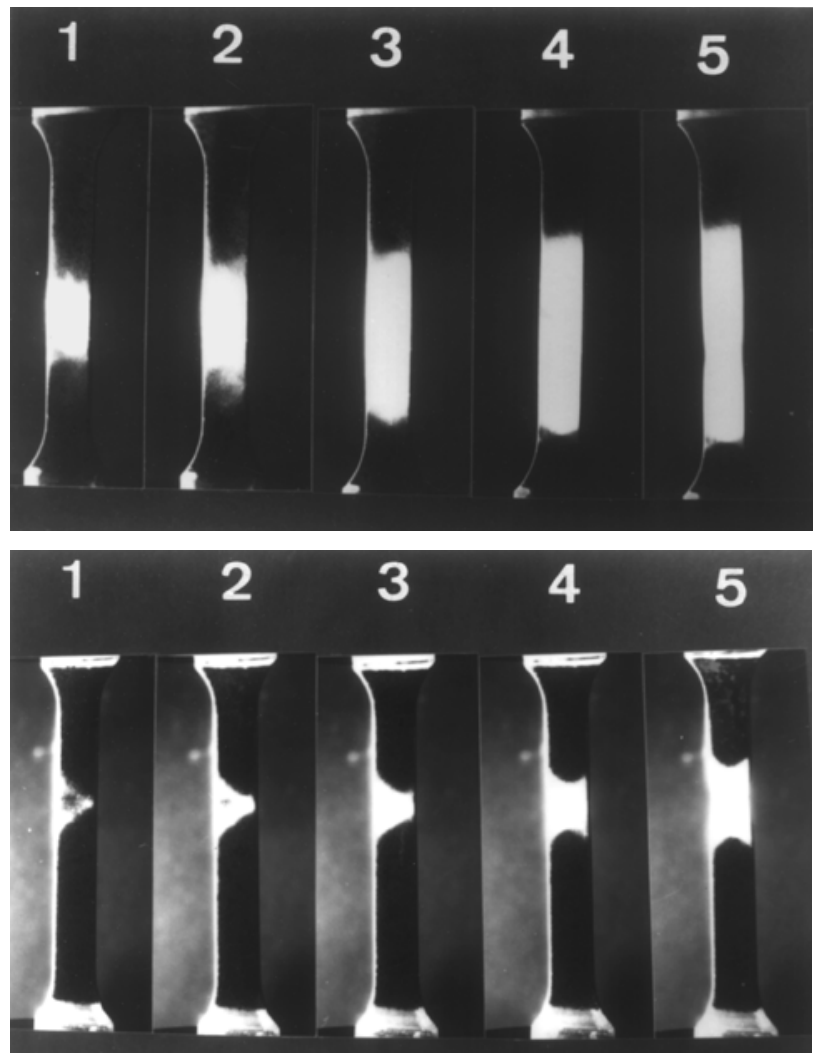

Figure 8. Evolution of the deformed region in tension after prestrain in rolling up to (a) $\varepsilon_{\mathrm{p}}=0.08$ and (b) $\varepsilon_{\mathrm{p}}=0.22$.

\begin{tabular}{lcc}
\multicolumn{3}{c}{ nominal strain } \\
No. & $8(\mathrm{a})$ & $8(\mathrm{~b})$ \\
1 & 0.04 & 0.02 \\
2 & 0.06 & 0.03 \\
3 & 0.10 & 0.04 \\
4 & 0.14 & 0.05 \\
5 & 0.28 & 0.07
\end{tabular}

than $20 \%$ of the total shear, in at least one set of Taylor solutions. The change in the active slip systems, which occurs after reloading, is thus easily seen. The most active slip systems during prestrain and during subsequent tension are different and, in most cases, the occurrence of a new slip system corresponds to the slip of dislocations with a Burgers vector, which has not been activated during prestrain. So, the yield stress increases after path change can be due to the latent hardening effect.

The relatively low work-hardening rate observed during the early stage of reloading can also be explained by the microstructural evolution induced by the change of slip systems. In fact, this behaviour is a result of two phenomena: the rapid increase of mobile dislocation density on the new active slip systems, most of them with a new Burgers vector, and the decrease in forest dislocation density due to a strong dynamic recovery of the previous structure.

TEM observations have shown mainly the presence of closed equiaxed cell structure, whatever the strain path in rolling or in tension with or without prestrain. For so small grain size (about $7 \mu \mathrm{m}$ ), the strain accommodation process is complex: the grains are strongly influenced by their surroundings and three or more non-coplanar systems have equivalent activities. This indicates that, for fine-grain material, the intergranular interaction approaches the Taylor condition of homogeneous deformation ${ }^{11}$.

Moreover, the plastic strain inside the grains is stable, i.e. no localised shear associated with microbands or any others instabilities have been observed, even after prestrain, contrary to that observed for large grain size as has been reported for copper ${ }^{7}$. Also, no shear offsets were noted in the grain boundary regions: the operating of supplemental systems in this region can satisfy the compatibility conditions near the grain boundary ${ }^{12}$.

The surface relief structure (Fig. 7) revealed closed active parallel slip planes. The orientation of slip lines in the grains does not depend on the observed region. Most of them are inclined to the tensile axes at an angle between 30 and $90^{\circ}$, as has already been observed on copper sheets with

Table 1. Sets of active slip systems according to the Taylor model in rolling and tension (normal to rolling), for three different grain orientations corresponding to the main texture components.

\begin{tabular}{|c|c|c|c|c|c|c|c|}
\hline Case & Texture component & \multicolumn{2}{|c|}{ Rolling } & \multicolumn{4}{|c|}{ Tension } \\
\hline 1 & $\left\{\begin{array}{lll}1 & 2 & 3\end{array}\right\}<3 \overline{3} 1>$ & $\overline{\mathrm{A} 3}$ & $\mathrm{C} 3$ & & & $\frac{\overline{4}}{2}$ & $\begin{array}{l}\text { D6 } \\
\text { D4 }\end{array}$ \\
\hline 2 & $\{112\}<1 \overline{1} 0>$ & $\overline{\mathrm{A} 3}$ & $\overline{\mathrm{D} 1}$ & & & $\frac{4}{2}$ & $\begin{array}{l}\text { D6 } \\
\text { D4 }\end{array}$ \\
\hline 3 & $\{110\}<1$ T $0>$ & $\begin{array}{l}\overline{\mathrm{A} 2} \\
\overline{\mathrm{A} 3} \\
\mathrm{~B} 2 \\
\mathrm{C} 3\end{array}$ & $\begin{array}{l}\mathrm{C} 1 \\
\mathrm{~B} 4 \\
\overline{\mathrm{D} 1} \\
\overline{\mathrm{D} 4}\end{array}$ & $\begin{array}{l}\text { A2 } \\
\text { A2 } \\
\text { A2 } \\
\text { A3 } \\
\text { A3 } \\
\text { B4 }\end{array}$ & $\begin{array}{l}\text { A3 } \\
\text { B2 } \\
\text { B4 } \\
\text { B2 } \\
\text { C1 } \\
\text { C1 }\end{array}$ & $\begin{array}{l}\mathrm{B} 2 \\
\mathrm{~B} 4 \\
\mathrm{C} 3 \\
\mathrm{C} 1 \\
\mathrm{C} 3 \\
\mathrm{D} 1\end{array}$ & $\begin{array}{l}\mathrm{C} 3 \\
\mathrm{D} 4 \\
\mathrm{D} 1 \\
\mathrm{D} 4 \\
\mathrm{D} 1 \\
\mathrm{D} 4\end{array}$ \\
\hline
\end{tabular}


$20 \mu \mathrm{m}$ grain size ${ }^{9}$. In particular, this kind of slip line distribution is noted inside the necking region, which confirms that there is no special configuration in the region of strain localisation.

Macroscopic shear bands that develop during rolling are sometimes associated with the appearance of plastic instability ${ }^{13}$. In copper, these shear bands have been observed only for rolling deformations higher than 0.5 . This is in accordance with our surface observations that indicate that no shear bands have been observed for the rolling deformation levels that we use in this work.

From the above analysis, it can be concluded that the appearance of plastic instability in tension of prestrained samples seems not to be directly controlled by the local substructure changes and must be associated with macroscopic plastic instabilities.

The results concerning macroscopic strain distribution show that the observed deformation can occur in a limited region in the sample which spreads out, before the final localisation of deformation takes place. This non-uniform deformation is surely connected with fluctuations in the area of the cross-section of the sample, due to rolling and subsequent machining, and with the mechanical properties after prestrain. This was confirmed using a simplified macroscopic two-zone model that allowed a qualitative study of the strain distribution in prestrained materials having a geometrical defect ${ }^{14}$. After path change, it was concluded that the non-uniform deformation at the beginning of reloading strongly depends on the work-hardening rate and yield stress values.

\section{Conclusion}

The present work examines the microstructural aspects and the mechanical behaviour of polycrystalline tensile specimens prestrained in rolling. The correlation established between the microscopic and macroscopic behaviour after the path change indicates that there is no apparent connection between non-homogeneous deformation at macroscopic level and slip behaviour inside the grains. In fact no local instabilities such as microbands or shear offsets at the grain boundaries were observed. The non-uni- form deformation clearly observed at the beginning of reloading in tension for prestrain values of 0.06 and higher is only connected with the plastic behaviour of prestrained samples.

\section{Acknowledgment}

The authors are indebted to FCT for financial support through FEDER and PRAXIS XXI Program.

\section{References}

1. Ghosh, A.K.; Backofen, W.A. Metall. Trans., v. 4, p. 1113, 1973.

2. Wagoner, R.H.; Laukonis, J.V. Metall. Trans. A, v. 14, p. 1487, 1983.

3. Chung, K.; Wagoner, R.H. Metall. Trans. A, v. 17, p. 1001, 1986.

4. Sharp, J.V.; Makin, M.J. Can. J. Phys., v. 45, p. 519, 1967.

5. Rauch, E.F.; Schmitt, J.-H. Mater. Sci. Eng. A, v. 113, p. 441, 1989.

6. Fernandes, J.V.; Gracio, J.J.; Schmitt, J.-H.; Rauch, E.F. Scr. Metall. Mater., v. 28, p. 1335, 1993.

7. Rao, B.V.N.; Laukonis, J.V. Mater. Sci. Eng., v. 60, p. $125,1983$.

8. Christodoulou, N.; Woo, O.T.; MacEwen, S.R. Acta Metall., v. 34, p. 1553, 1986.

9. Schmitt, J.-H.; Fernandes, J.V.; Gracio, J.J.; Vieira, M.F. Mater. Sci. Eng. A, v. 147, p. 143, 1991.

10. Vieira, M.F.; Schmitt, J.-H.; Gracio, J.J.; Fernandes, J.V. J. Mater. Proc. Tech., v. 24, p. 313, 1990.

11. Gracio, J.J.; Fernandes, J.V.; Schmitt, J.-H. Mater. Sci. Eng. A, v. 118, p. 97, 1989.

12. Kocks, U.F.; Canova, G.R. Deformation of Polycrystals: Mechanisms and Microstructures, eds. N. Hansen et al., Riso National Laboratory, Roskilde, p. 35-44, 1981.

13. Malin, A.S.; Hatherly, M. Metal Science, v. 137, p. 463, 1979.

14. Fernandes, J.V.; Vieira, M.F. Metall. Mater. Trans., v. 28A, p. 1169, 1997. 